\title{
Hemi-ovariossalpingohisterectomia em pacas prenhes e posterior ocorrência de prenhez (Agouti paca, Linnaeus, 1766)
}

\author{
Hemiovarysalpingohysterectomy in pregnant pacas and further occurrency of pregnancy \\ (Agouti paca, Linnaeus, 1766)
}

\author{
Fabrício Singaretti de Oliveira ${ }^{1}$ Gilson Hélio Toniollo ${ }^{2}$ \\ Márcia Rita Fernandes Machado ${ }^{3}$ Daniela Paura $^{4}$
}

RESUMO

Foi descrita a hemi-ovariossalpingohisterectomia em cinco pacas prenhes mantidas em cativeiro no Setor de Animais Silvestres da Faculdade de Ciências Agrárias e Veterinárias (FCAV-UNESP) de Jaboticabal, São Paulo, Brasil, a fim de observar futura prenhez no corno restante. A tranqüilização foi obtida após aplicação de azaperone (4mg) $\mathrm{kg})$ seguida da aplicação de sulfato de atropina $(0,06 \mathrm{mg} / \mathrm{kg})$ $e$ da associação de cloridrato de quetamina (20 $\mathrm{mg} / \mathrm{kg})$ e cloridrato de xilazina $(1,5 \mathrm{mg} / \mathrm{kg})$, ambos na mesma seringa, para indução da anestesia. A anestesia geral foi obtida mediante inalação de halotano por máscara. Por meio de laparotomia mediana, foram retirados o corno uterino prenhe, o ovário e a tuba uterina, todos do mesmo antímero. Antibióticos (30.000UI/ $\mathrm{kg}$ de três penicilinas e $12,5 \mathrm{mg} / \mathrm{kg}$ de duas estreptomicinas) $e$ analgésico $(0,02 \mathrm{mg} / \mathrm{kg}$ de buprenorfina) foram aplicados imediatamente após a cirurgia, sendo repetidos após dois dias. Todas as aplicações foram feitas por via intramuscular. Apesar da permanência de apenas um ovário após a cirurgia, nova prenhez ocorreu no corno restante nas cinco fêmeas submetidas à cirurgia, com o nascimento de filhotes (apenas um por parto) após 215, 248, 276, 302 e 310 dias da hemiovariossalpingohisterectomia.

Palavras-chave: Agouti paca, cirurgia, prenhez, anestesia.

\section{ABSTRACT}

The hemiovarysalpingohysterectomy in five captive pregnant pacas kept on the Wild Animal Section at the College of Agricultural and Veterinarian Sciences (FCAV-
UNESP) in Jaboticabal, São Paulo, Brazil, was conducted aiming at verifying the further occurrence of pregnancy on the remaining uterine horn. The tranquilization was achieved by using azaperone $(4 \mathrm{mg} / \mathrm{kg})$ and consequent application of atropine sulphate $(0.06 \mathrm{mg} / \mathrm{kg})$ and the association of ketamine $(20 \mathrm{mg} / \mathrm{kg})$ and xylazine $(1.5 \mathrm{mg} / \mathrm{kg})$ cloridrates, on the same syringe, for induction of anesthesia. The general anesthesia was performed via a face mask with halothane. By means of median laparotomy, the uterine horn with the fetus, the ovary and the uterine tube, all from the same antimere, were taken out. Antibiotics $(30,000 I \mathrm{U} / \mathrm{kg}$ of three penicillins and $12.5 \mathrm{mg} /$ $\mathrm{kg}$ of two streptomycins) and analgesic (buprenorfine 0.02 $\mathrm{mg} / \mathrm{kg}$ ) were injected immediately after surgery and repeated after two days. All injections were made intramuscularly. In spite of the permanence of only one ovary after surgery, new pregnancy occurred in the remaining uterine horn in the five females which went through surgery, and the birth (solely one by delivery) happened 210,248, 276, 302 and 310 days after the hemiovarysalpingohysterectomy.

Key words: Agouti paca, surgery, pregnancy, anesthesia.

\section{INTRODUÇÃO}

Depois da capivara, a paca é o maior roedor da região neotropical (MONDOLFI, 1972; MATAMOROS, 1982; SILVA, 1984); os machos adultos medem de 60 a 80 centímetros, do focinho à ponta da cauda, e as fêmeas, de 55 a 70 centímetros, de acordo

${ }^{1}$ Aluno do Curso de Pós-graduação em Cirurgia Veterinária, Nível Doutorado, Faculdade de Ciências Agrárias e Veterinárias (FCAV),

UNESP, Jaboticabal, SP. Via de acesso Prof. Paulo Donato Castelani, s/n. Depto de Clínica e Cirurgia Veterinária, $14884-900$. Jaboticabal, SP. E-mail: singaretti@ig.com.br. Autor para correspondência.

${ }^{2}$ Professor Adjunto, Departamento de Medicina Veterinária Preventiva e Reprodução Animal, FCAV, UNESP.

${ }^{3}$ Professor Adjunto, Doutor, Departamento de Morfologia e Fisiologia Animal, FCAV, UNESP.

${ }^{4}$ Médico Veterinário Aprimorando em Reprodução e Obstetrícia Veterinária, FCAV, UNESP. 
com MONDOLFI (1972) e BENTTI (1981). O peso corpóreo varia de 5 a $10 \mathrm{~kg}$, podendo chegar até aos 14 $\mathrm{kg}$, de acordo com MATAMOROS (1982), mas não ultrapassa $10 \mathrm{~kg}$ como relataram DEUTSCH \& PUGLIA (1988).

A paca é um animal com corpo robusto e vigoroso, especialmente a garupa e os membros pélvicos, notadamente musculosos (VIEIRA, 1953; BENTTI, 1981; COLLET, 1981; SILVA, 1984) e é encontrada desde o sudeste do México até o norte do Paraguai, em altitudes de até 3.000 metros (DEUTSH \& PUGLIA, 1988; EMMONS, 1990), adaptando-se bem a ambientes muito variados e preferindo as zonas cobertas com vegetação alta, vizinhas a rios ou riachos (DEUTSH \& PUGLIA, 1988).

Quanto ao período de prenhez desses animais, há diferentes citações na literatura. Este período não ultrapassaria 115 dias de acordo com PÉREZ \& HERNANDES (1979), KLEIMAN et al. (1979) e CLARK \& OLFERT (1986). Já mediante as citações de COLLET (1981), não excederia 116 dias. Para MATAMOROS \& PASHOV (1984) o período abrange 114,28 dias, em média, com variação de 85 a 156 dias, e para SMYTHE (1991), é em torno de 155 dias, variando de 138 a 173 dias. NOGUEIRA (1997) citou um período de prenhez de aproximadamente 150 dias, enquanto OLIVEIRA (2002), em pesquisa utilizando a ultrasonografia para determinação do período de prenhez, concluiu que o mesmo abrange 135 a 139 dias nessa espécie.

MONDOLFI(1972), PÉREZ \& HERNANDES (1979), BENTTI (1981) e MATAMOROS (1982) citaram que, geralmente, nasce um filhote por parto e, dificilmente, dois ou três. $\mathrm{O}$ ciclo reprodutivo da fêmea é de 28 a 31 dias e o ciclo estral pode ocorrer logo após o nascimento e, novamente, após o desmame. Após 28 dias do nascimento, ocorre um novo ciclo estral pósparto (HOSKEN, 1999).

A paca é um animal de útero bicórneo e cada corno mede, aproximadamente, $12 \mathrm{~cm}$ de comprimento, sendo unidos externamente por meio de uma fina membrana, que forma um falso corpo. Os ovários estão localizados caudalmente aos rins e são amarelados, ovalados e lisos, com cerca de $0,8 \mathrm{~cm}$ de comprimento e $0,5 \mathrm{~cm}$ de largura. As tubas uterinas são finas, contorcidas, medem, aproximadamente, $5 \mathrm{~cm}$ de comprimento e estão em contato com a superfície medial dos ovários (MATAMOROS, 1981).

BJÖRKMAN et al. (1989) relataram que os roedores, por apresentarem aspectos característicos, tais como tamanho adequado, baixo custo de manutenção e curto período de prenhez, são considerados animais experimentais " $a d$ hoc" embora ainda faltem informações precisas sobre aspectos reprodutivos de algumas espécies, o que gera, muitas vezes, interpretações errôneas de experimentos. Neste contexto, HAMELETT \& RASWEILER IV (1993) salientaram a importância da busca de novas espécies animais com potencial para serem utilizadas como modelos experimentais, colaborando com o desenvolvimento de pesquisas vitais ao homem e aos próprios animais.

A castração é, certamente, o melhor método de controle populacional de animais domésticos e o mais empregado na rotina veterinária, pois, além de evitar o sacrifício em massa de animais, impede os riscos de doenças que ocorrem com o uso de drogas anticoncepcionais (FINGLAND, 1996; STONE, 1998). Em camundongos, a hemi-histerectomia, durante a prenhez, provocou maior peso dos neonatos quando em relação ao grupo controle, não afetando a mortalidade fetal (KNIGHT \& PEAKER, 1982)

Hemi-castrações para colheita de placenta, do funículo umbilical, do feto ou do ovário podem fornecer importantes informações sobre o ciclo reprodutivo, o qual é pouco estudado em animais selvagens. Nesses, pelo fato da procriação ser de extrema importância para a preservação das espécies, esse tipo de cirurgia pode ser indicada por não implicar na perda da capacidade reprodutiva da fêmea.

O objetivo deste trabalho foi avaliar se, em pacas submetidas à hemi-ovariossalpingohisterectomia, ocorre um comprometimento de futura prenhez. A carência de informações sobre a continuidade da vida reprodutiva de animais selvagens submetidos à hemiovariossalpingohisterectomia, aliada à importância de sua procriação, preservação e do seu potencial de atuação como modelo experimental, justifica a realização dessa pesquisa.

\section{MATERIALE MÉTODOS}

Para a realização desta pesquisa, foram utilizadas cinco pacas fêmeas prenhes, mantidas em cativeiro no Setor de Animais Silvestres do Departamento de Zootecnia da Faculdade de Ciências Agrárias e Veterinárias, Campus de Jaboticabal as quais foram submetidas à hemi-ovariossalpingohisterectomia devido à necessidade de colheita da placenta, do funículo umbilical, do feto e de um ovário para pesquisa.

Os animais foram alojados em baias de aproximadamente $15 \mathrm{~m}^{2}$ de alvenaria, com uma toca por baia, subdividida em três repartições internas comunicantes, permanecendo, em cada baia, um macho e duas ou três fêmeas com marcação individual por microchips, aplicados dorsalmente na região cervical. 
Na alimentação dos animais, foram utilizadas ração de roedores ${ }^{\mathrm{a}}(1,0 \%$ do peso vivo por dia) e frutas da época ( $10 \%$ do peso vivo por dia), oferecidas diariamente no período da tarde.

A cirurgia foi realizada imediatamente após confirmação de prenhez mediante sessão de ultrasonografia em modo B, usando-se transdutor bifreqüencial setorial eletrônico de 5,0 e 7,5 $\mathrm{MHz}^{\mathrm{b}}$ para visualização do feto em estádio intermediário ou avançado de desenvolvimento.

A aplicação de tranqüilizantes, anestésicos, analgésicos e antibióticos, nas pacas, foi realizada sempre por via intra-muscular, nos membros pélvicos. A tranqüilização foi obtida com azaperone ${ }^{c}$ na dose de $4 \mathrm{mg} / \mathrm{kg}$ de peso corporal. Após 10 minutos, aplicou-se $0,06 \mathrm{mg} / \mathrm{kg}$ sulfato de atropina ${ }^{\mathrm{d}}$, seguindo-se, após mesmo intervalo, a administração da associação de cloridratos de quetamina $^{e}(20 \mathrm{mg} / \mathrm{kg})$ e de xilazina ${ }^{\mathrm{f}}$ $(1,5 \mathrm{mg} / \mathrm{kg})$ para indução da anestesia. Realizou-se tricotomia abdominal ampla com lâmina de aço inoxidável e a anestesia geral foi obtida mediante inalação de halotano ${ }^{\mathrm{g}}$ por máscara, fornecido juntamente com $750 \mathrm{~cm}^{3}$ de oxigênio puro por minuto.

A incisão da pele foi feita na linha média, sentido pré-retroumbilical, abrangendo cerca de 12 centímetros, devido ao considerável volume do corno uterino prenhe. O ovário foi identificado e, com o auxílio de duas pinças dente de rato colocadas no pedículo ovariano a um centímetro uma da outra, foi mantido suspenso e exteriorizado mediante incisão, procedendose, então, sua ligadura. Duas outras pinças, iguais às anteriores, foram colocadas na porção inicial do corno uterino prenhe, incidindo-se entre as mesmas e removendo-se o corno em questão. Suturas tipo Schmieden (primeira plano) e tipo Cushing (segundo plano) foram realizadas no útero, colocando-se o epíplon sobre a mesma ao final desse procedimento. A camada muscular foi suturada utilizando-se ponto em $X$. Nas suturas do pedículo ovariano, do útero e da camada muscular foi utilizado fio categute cromado número $2^{\mathrm{h}} \mathrm{e}$, na sutura da pele, foi utilizado fio de náilon monofilamentado número $0^{\mathrm{h}}$.

Imediatamente, após a cirurgia, foi realizado curativo local com solução de iodo a $2 \%$, colocado esparadrapo hipoalergênico ${ }^{\mathrm{i}}$ sobre a sutura de pele e aplicados antibióticos ${ }^{\mathrm{j}}(15.000 \mathrm{U} / \mathrm{kg}$ de benzilpenicilina benzatina, $7.500 \mathrm{U} / \mathrm{kg}$ de benzilpenicilina procaína, $7.500 \mathrm{U} / \mathrm{kg}$ de benzilpenicilina potássica, $6,25 \mathrm{mg} / \mathrm{kg}$ de sulfato de diidroestreptomicina e $6,25 \mathrm{mg} / \mathrm{kg}$ de sulfato de estreptomicina) e $0,020 \mathrm{mg} / \mathrm{kg}$ de buprenorfina ${ }^{1}$ para analgesia. Depois de 48 horas, outra dose dos antibióticos foi administrada e o esparadrapo mantido até a retirada dos pontos da pele, ocorrida após sete dias da cirurgia.

\section{RESULTADOS E DISCUSSÃO}

A tranqüilização das pacas mostrou-se eficiente, apesar do emprego do azaperone, conhecido como um tranqüilizante específico para suínos. Após 10 minutos da administração desse princípio ativo, as fêmeas apresentavam-se calmas e indiferentes ao meio. As quantidades administradas dos cloridratos de quetamina e de xilazina foram suficientes apenas para indução da anestesia, enquanto a analgesia necessária para o procedimento cirúrgico foi obtida somente mediante inalação de halotano por máscara. Houve bom miorrelaxamento e as cirurgias demoraram de 30 a 40 minutos, não ocorrendo problemas nos períodos pré, trans ou pós-operatório.

As cinco fêmeas submetidas à retirada do corno uterino prenhe, da tuba uterina e do ovário, todos no mesmo antímero, apresentaram posterior prenhez no corno restante e o nascimento dos filhotes ocorreu entre 215 e 310 dias após a cirurgia (Tabela 1).

Considerando-se 115 dias a duração da prenhez na paca (PÉREZ \& HERNANDES, 1979; KLEIMAN et al., 1979 e CLARK \& OLFERT, 1986), a prenhez seguinte à retirada do corno uterino com o feto ocorreu entre 100 e 195 dias da cirurgia. Esses valores aumentariam em um dia, levando-se em conta um período de 116 dias (COLLET, 1981) ou diminuiriam praticamente um dia, considerando-se 114,28 dias a duração da prenhez nesses animais (MATAMOROS \& PASHOV, 1984).

Se forem considerados os períodos propostos por NOGUEIRA (1997), de 150 dias, e por SMYTHE (1991), de 155 dias, a prenhez ocorreu depois de 65 a 160 dias e de 60 a 155 dias da cirurgia para retirada do corno uterino prenhe, respectivamente.

Tabela 1 - Identificação das pacas submetidas à hemiovariossalpingohisterectomia do corno prenhe posterior ocorrência de prenhez no corno restante.

\begin{tabular}{ccc}
\hline $\begin{array}{c}\text { Código do } \\
\text { microchipda } \\
\text { fêmea prenhe }\end{array}$ & $\begin{array}{c}\text { Data da } \\
\text { cirurgia }\end{array}$ & $\begin{array}{c}\text { Nascimento do filhote } \\
\text { (dias após a cirurgia) }\end{array}$ \\
\hline 00-02-05-07-E2T & $06 / 03 / 2000$ & 215 \\
00-01-F0-05-40T & $08 / 05 / 2000$ & 302 \\
00-01-F0-6E-CAT & $21 / 08 / 2000$ & 310 \\
00-01-C7-E0-5AT & $04 / 12 / 2000$ & 248 \\
$00-01-\mathrm{F} 0-7 \mathrm{C}-41 \mathrm{~T}$ & $04 / 12 / 2000$ & 276 \\
\hline
\end{tabular}


Em relação ao período de 135 a 139 dias determinado por meio de ultra-sonografia por OLIVEIRA (2002), novas prenhezes ocorreram entre 76 e 175 dias após a hemi-ovariossalpingohisterectomia, ou seja, mesmo após a retirada cirúrgica de um ovário, o ciclo reprodutivo das fêmeas voltou a ocorrer rapidamente. Isso levou a ocorrência de novos partos entre 215 e 310 dias da cirurgia, o que é similar às citações de HOSKEN (1997), o qual relatou a ocorrência de ciclos estrais logo após o parto e o desmame.

Ocorreu o nascimento de apenas um filhote por parto, o que está de acordo com as descrições de MONDOLFI (1972), PÉREZ \& HERNANDES (1979), BENTTI (1981) e MATAMOROS (1982).

A hemi-ovariossalpingohisterectomia, realizada nas pacas prenhes desta pesquisa, não impediu a continuidade da vida reprodutiva desses animais, demonstrada pelo nascimento dos filhotes gerados no corno uterino restante. Assim, nesses roedores, podem ser conduzidas pesquisas que necessitem da remoção cirúrgica do corno uterino prenhe e do ovário do mesmo antímero sem haver o comprometimento de futuras prenhezes, o que é muito importante para preservação da espécie.

Desde que corretamente realizada, a hemiovariossalpingohisterectomia pode ser empregada para a colheita de estruturas necessárias a pesquisas que venham a fornecer subsídios para interpretações corretas de experimentos relativos ao homem e a outras espécies animais.

\section{FONTES DE AQUISIÇÃO}

\author{
a Labina - Agribrands do Brasil Ltda. \\ bPie Medical ${ }^{\circledR}$ LC 100 Vet Ltda \\ 'Stresnil ${ }^{\circledR}$ - Janssen Pharmaceutica \\ 'Sulfato de atropina ${ }^{\circledR}$ - Ariston Ltda. \\ 'Ketamina 50® - Holliday-Scott S.A \\ ${ }^{\mathrm{f} C}$ Coopazine ${ }^{\circledR}$ - Coopers Brasil Ltda. \\ ${ }^{g}$ Halotano ${ }^{\circledR}$ - Cristália Produtos Químicos Farmacêuticos Ltda \\ ${ }^{\text {hEthicon}}{ }^{\circledR}$ Ltda \\ ${ }^{\mathrm{i}}$ Micropore ${ }^{\circledR}$ - $3 \mathrm{M}$ do Brasil Ltda \\ jPentabiótico Veterinário ${ }^{\circledR}$ - Fort Dodge Saúde Animal Ltda \\ ${ }^{1}$ Temgesic ${ }^{\circledR}$ - Schering-Plough Ltda.
}

\section{REFERÊNCIASBIBLIOGRÁFICAS}

BENTTI, S.B. Roedores da américa tropical. Natura, Caracas, n. $70-1$, p.40-4, 1981.

BJÖRKMAN, N.; DANTZER, V.; LEISER, R. Comparative placentation in laboratory animals-a review. Scandinavian Journal of Animal Science, v.16, n.4, p.129-158, 1989.

CLARK, J.D.; OLFERT, E.D. Rodents (Rodentia). In: FOWLER, M.E. Zoo \& Wild animal medicine. 2.ed. Philadelphia: Saunders, 1986. p.728-737.
COLLET, S.F. Population characteristics of agouti paca (rodentia) in colombia. Biological Series, Michigan, v.5, n.7, p.601, 1981 .

DEUTSCH, L.A.; PUGLIA, L.R.R. Paca: os animais silvestres: proteção, doenças e manejo. Rio de Janeiro: Globo, 1988. p.45-50.

EMMONS, L.H. Paca. In: Neotropical rainforest mammals: a field guide. Chicago: The University of Chicago, 1990. p.204-205.

FINGLAND, R.B. Castração em pequenos animais. In: BOJRAB, M.J. Técnicas atuais em cirurgia de pequenos animais. 3.ed. São Paulo : Roca, 1996. p.375-384.

HAMELETT, W.C.; RASWEILER IV, J.J. Comparative gestation and placentation in vertebrates. The Journal of Experimental Zoology, v.266, p.343-346, 1993.

HOSKEN, F.M. Criação de pacas. Cuiabá: Sebrae/MT, 1999. p.25-26. (Série Natureza e Negócios, 2).

KLEIMAN, D.G.; EISENBERG，J.F.; MALINIAK, E. Reproductive parameters and productivity of caviomorph rodents. In: EISENBERG, J.F. Vertebrate ecology in the northern neotropics. Washington : Smithsonian Institution, 1979. p.173-183.

KNIGHT, C.H.; PEAKER, M. Mammary development in mice: effects of hemihysterectomy in pregnancy and of litter size post partum. Journal of Physiology, Cambridge, v.327, p.1727, 1982 .

MATAMOROS, Y. Anatomía e histología del sistema reproductor del tepezcuinte (Cuniculus paca). Revista de Biología Tropical. v.29, n.1, p.155-164, 1981.

MATAMOROS, Y. Notas sobre la biologia del tepezcuinte, Cuniculus paca, brisson, (Rodentia: Dasyproctidae) en cautiverio. Brenesia, San Jose, n.19/20, p.71-82, 1982.

MATAMOROS, Y.; PASHOV, B. Ciclo estral del tepezcuinte (Cuniculus paca, Brisson) en cautiverio. Brenesia, San Jose, n.22, p.249-260, 1984.

MONDOLFI, E. La laca o paca. Defensa de la naturaleza, Caracas, v.2, n.5, p.4-16, 1972.

NOGUEIRA, T.M.R. Alguns parâmetros fisiológicos e reprodutivos da paca (Agouti paca, Linnaeus, 1766), em cativeiro. 1997. 131f. Dissertação (Mestrado em Produção Animal) - Curso de Pós-graduação em Zootecnia, Faculdade de Ciências Agrárias e Veterinárias de Jaboticabal, Universidade Estadual Paulista.

OLIVEIRA, F.S. Diagnóstico e acompanhamento ultrasonográfico da prenhez em pacas (Agouti paca, Linnaeus, 1766). 2002. 45f. Dissertação (Mestrado em Cirurgia Veterinária) - Curso de Pós-graduação em Cirurgia Veterinária, Faculdade de Ciências Agrárias e Veterinárias de Jaboticabal, Universidade Estadual Paulista.

PÉREZ, H.C.; HERNANDEZ, F.Z. Comportamiento reproductivo y datos de la alimentación de Agouti paca nelsoni. Acta Zoologica Lilloana, San Miguel de Tucumán, v.35, p.283285,1979 
SILVA, F. Ordem Rodentia: mamíferos silvestres do Rio Grande do Sul. Porto Alegre : Fundação Zoobotânica do Rio Grande do Sul, 1984. p.171-209.

SMYTHE, N. Paca. In ROBINSON, J.G.; REDFORD, K.H. Microlivestock: little known small animals with promising economic future. Washington : National Academy, 1991. p.263-269.
STONE, E.A. Ovariohisterectomia. In: SLATTER, D. Manual de cirurgia de pequenos animais. 2.ed. Philadelphia: Saunders, 1998. V.2, p.1552-1554.

VIEIRA, C. Roedores e lagomorfos do estado de São Paulo. Arquivos de Zoologia, São Paulo, v.8, p.129160,1953 